\title{
Multiring electrostatic guide for Rydberg positronium
}

\author{
M. H. Rayment, L. Gurung, R. E. Sheldon, S. D. Hogan, and D. B. Cassidy \\ Department of Physics and Astronomy, University College London, Gower Street, London WC1E 6BT, United Kingdom
}

(Received 16 May 2019; published 15 July 2019)

\begin{abstract}
We report the results of experiments in which positronium (Ps) atoms, optically excited to Rydberg-Stark states with principal quantum numbers ranging from $n=13$ to 19 , were transported along the axis of a multiring electrode structure. By applying alternate positive and negative potentials to the ring electrodes, inhomogeneous electric fields suitable for guiding low-field-seeking atoms along the guide axis were generated. The multiring configuration used has the advantage that once the atoms are confined within it appropriate time-varying fields can be generated for deceleration and trapping. However, in this type of structure the possibility of nonadiabatic transitions of the fast $(100 \mathrm{~km} / \mathrm{s})$ Ps atoms to unconfined high-field-seeking states exists. We show that for typical guiding fields this is not a significant loss mechanism and that efficient Ps transport can be achieved. Our data are in accordance with a Landau-Zener analysis of adiabatic transport through the field minima and Monte Carlo simulations that take into account Ps velocity distributions, electric dipole moments, and lifetimes, as well as the electric-field distributions in the guide.
\end{abstract}

DOI: 10.1103/PhysRevA.100.013410

\section{INTRODUCTION}

As a low-mass matter-antimatter hydrogenic atomic system composed only of leptons, positronium (Ps) is, for all practical purposes [1], fully described by bound-state QED theory [2]; as such, Ps is an ideal system with which to test this theory. Such tests can also be used to search for new, low-mass, particles that may not be included in the standard model (SM) [3,4], since any verified deviations from QED predictions must then arise from non-SM effects. In fact, precision spectroscopy of atomic and molecular systems is increasingly being exploited as a way to test for new physics at the low-energy frontier [5], in contrast to measurements at the high-energy frontier, as exemplified by the Large Hadron Collider [6,7]. In principle Ps spectroscopy could play a unique role in such research, but this will only be possible if certain experimental limitations related to the formation of cold Ps gases can be overcome [8].

The production of low-energy (i.e., $<1 \mathrm{eV}$ ) positronium atoms usually begins with the bombardment of a solid target by energetic (i.e., $>100 \mathrm{eV}$ ) positrons. Then, depending on the nature of the target [9-11], one of several possible mechanisms may result in the generation of near-thermal Ps atoms in vacuum. However, the low mass of Ps means that thermal atoms have speeds on the order of $10^{5} \mathrm{~ms}^{-1}$, which is much faster than atomic beams produced, for example, by supersonic expansion [12] and introduces several complications to Ps experiments.

The annihilation rate of Ps atoms depends on their principal quantum number, spin, and orbital angular momentum (e.g., Ref. [13]). The mean annihilation lifetime of singlet (triplet) states with $\ell=0$ is $0.125 \times n^{3}$ ns [14] $\left(142 \times n^{3}\right.$ ns [15]). Thus thermal Ps atoms in the ground state will travel no more than a few $\mathrm{cm}$ before self-annihilation. Moreover, the angular distributions of low energy Ps are often rather broad, meaning that beam formation may require collimation and a concomitant loss of flux. It is therefore advantageous for some Ps experiments, in particular precision spectroscopy [16], to control both the motion and lifetimes of Ps atoms; both of these goals can be accomplished by exciting them to Rydberg states [17].

The electron-positron wave-function overlap, and hence annihilation rate, is negligible for Ps states with angular momentum $\ell \neq 0[18,19]$. Due to the intrinsic $\ell$ degeneracy of hydrogenic atoms [20], even very small electric fields, which are notoriously difficult to eliminate [21], can lead to complete $\ell$ mixing for Rydberg states [22]. As a result, the lifetimes of Ps atoms in $\ell$-mixed Rydberg-Stark states are almost entirely determined by fluorescence decay rates which are approximately half those of the corresponding levels in hydrogen and scale with $\approx n^{4}$ [23].

Ps Rydberg-Stark states may also possess large static electric dipole moments [24], which can be employed to control the motion of the atoms using inhomogeneous electric fields [25-27]. This was first demonstrated by deflecting beams of Rydberg $\mathrm{Kr}$ atoms [28]; subsequently, various atom optics elements have been developed, such as mirrors [29], lenses [30], deflectors [31,32], and decelerators and traps [33-36].

Deceleration and trapping of Rydberg Ps has not yet been achieved, but electrostatic guiding has been previously demonstrated using quadrupolar electric fields [37,38]. An electrostatic mirror has also been developed that can focus a Rydberg Ps beam over a distance of 6 m [39]. Here we report the development of a multiring device that can be used to confine and guide low-field-seeking atoms in two dimensions. We show that, despite Ps atoms traversing regions of zero electric field as they travel through this device, no significant losses are observed as a result of nonadiabatic transitions. The multiring electrode configuration used here allows for better control over Ps trajectories than in a quadrupole guide and can, in principle, be used to apply time-varying fields that decelerate and trap Rydberg atoms [27]. 


\section{EXPERIMENTAL PROCEDURE}

The positron trap used in this work is the same as that described in [40] and the detectors and measurement methods are detailed in [41]. The general methodology of the present experiments was as follows: a pulsed positron beam, compressed temporally and spatially to $2.7 \mathrm{~ns}$ and $1.3 \mathrm{~mm}$ (FWHM), respectively, was implanted at $1 \mathrm{~Hz}$ into a thin mesoporous silica target in order to produce Ps atoms [10,42]. The target electrode was mounted on a three-axis manipulator and could be moved out of the beam path to allow imaging on a microchannel plate (MCP) detector coupled to a phosphor screen. Approximately $10^{5}$ Ps atoms per pulse were emitted from the silica target within $10 \mathrm{~ns}$, and with energies on the order of $50 \mathrm{meV}$ [43], leading to the creation of a Ps gas in vacuum with an initial density on the order of $10^{7} \mathrm{~cm}^{-3}$.

Rydberg Ps atoms were generated using the two-step twocolor photoexcitation scheme first demonstrated by Ziock and co-workers [44]; a pulsed ultraviolet (UV) dye laser $(\approx 500 \mu \mathrm{J} /$ pulse, $\Delta v=85 \mathrm{GHz}, \lambda=243 \mathrm{~nm})$ was used to drive $1{ }^{3} S_{1} \rightarrow 2^{3} P_{J}$ transitions and a second synchronous infrared (IR) dye laser $(\approx 3 \mathrm{~mJ} /$ pulse, $\Delta v=5 \mathrm{GHz}, \lambda=$ $729-760 \mathrm{~nm}$ ) was used to drive $2^{3} P_{J} \rightarrow n S / n D$ transitions, or to photoionize $2^{3} P_{J}$ atoms [45]. Both the UV and IR laser beams were propagated parallel to the silica target surface (see Fig. 1). Both lasers were linearly polarized, with the UV and IR parallel and perpendicular to the positron beam axis, respectively. This resulted in the production of Rydberg Ps atoms with azimuthal quantum number $|m|=1$ [24].

The formation, excitation, and transport of Ps was monitored via the time dependence of its annihilation radiation, recorded by five $\gamma$-ray detectors placed outside the vacuum system at different locations around the Ps production region and guide electrodes, as indicated in Fig. 1. Each detector was made from a scintillator crystal optically coupled via a light guide to a photomultiplier tube (PMT). In this work lutetiumyttrium oxyorthosilicate (LYSO) scintillators were used [41]. The LYSO detectors have an intrinsic decay time of $\sim 40 \mathrm{~ns}$ and could be used to detect individual annihilation events [23], or to generate single-shot lifetime spectra comprising multiple near-simultaneous events [46].

Detector D1 was used to generate single-shot lifetime spectra from which Ps production was quantified using the parameter $f_{\mathrm{d}}$, which is proportional to the amount of o-Ps present and is defined as $f_{\mathrm{d}}=\int_{B}^{C} V(t) d t / \int_{A}^{C} V(t) d t$, where $V(t)$ is the detector output voltage. The time windows used to analyze lifetime spectra $(A, B, C)$ were $(-20,120,700 \mathrm{~ns})$, where time zero corresponds to the positron beam implantation time. Optical excitation of Ps atoms was characterized by the parameter $S_{\gamma}=\frac{f_{\mathrm{d}(\text { off }}-f_{\mathrm{d}(\text { on })}}{f_{\mathrm{d}(\mathrm{off})}}$, where $f_{\mathrm{d}(\text { on })}$ and $f_{\mathrm{d}(\text { off })}$ refer to measurements made with the excitation lasers on and off resonance [40].

Spectra of Doppler broadened $1{ }^{3} S_{1} \rightarrow 2{ }^{3} P_{J}$ transitions were used to determine the mean Ps velocity spread in the direction of the UV laser. Line shapes were fitted to $S_{\gamma}(\lambda)=$ $A \exp \frac{-\left(\lambda-\lambda_{0}\right)^{2}}{2 \sigma^{2}}$, where $\sigma$ and $\lambda_{0}$ are the Gaussian width and the resonance wavelength, respectively. The mean-squared Ps velocity along the laser direction is then $\left\langle v_{\mathrm{x}}^{2}\right\rangle=\left[c \sigma / \lambda_{0}\right]^{2}$

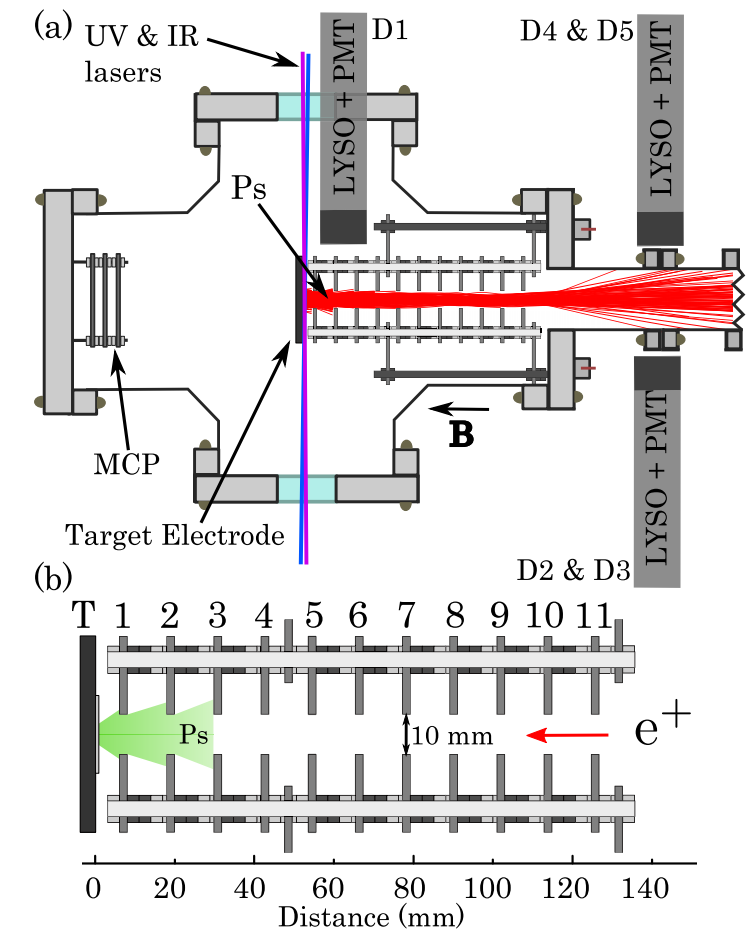

FIG. 1. (a) Schematic representation of the target chamber, multiring electrode structure, and detector layout. Multiple detectors were positioned perpendicular to the beam axis, not all of which are shown in the figure. (b) Layout of the multiring electrode structure showing the target electrode (T) and the guide electrodes (E1-E11). Ps atoms were generated in the silica target and lasers entered the excitation region between $\mathrm{T}$ and E1. E1-E4 were all independently controlled, with electrodes coupled in pairs thereafter (i.e., E5, E7, $\mathrm{E} 9$, and E11 were all connected as were E6, E8, and E10).

and the associated kinetic energy $E_{\mathrm{x}}=\frac{1}{2} m_{\mathrm{Ps}} v_{\mathrm{x}}^{2} \cdot S_{\gamma}$ was also used to characterize the measured $2{ }^{3} P_{J} \rightarrow 13{ }^{3} S / 13{ }^{3} D$ spectra.

TOF spectra were measured via single-event counting methods [23] using detectors D2-5 [see Fig. 1(a)]. Trigger events were determined by postprocessing the recorded LYSO waveforms, where an event was registered if the signal amplitude exceeded a threshold level of $-0.5 \mathrm{mV}$ for a period of at least $30 \mathrm{~ns}$. The arrival time of the event signal leading edge, as well as its amplitude and width, were recorded by an oscilloscope (500 MHz bandwidth, sampling rate $10 \mathrm{GSa} / \mathrm{s}$ ) and stored off line for every positron pulse.

As indicated in Fig. 1, Ps atoms were produced and excited in the region between the target electrode (T) and the first electrode of the guide (E1). These electrodes could be separately biased, making it possible to control both the positron beam implantation energy and the electric field in the excitation region independently. The former controls the kinetic energy of the emitted Ps [47]. The latter allows the Stark state degeneracy in zero electric field to be lifted and thus specific parts of the Stark manifold to be addressed by tuning the IR laser wavelength [24]. Specifically, in this way we may predominantly photoexcite Stark states with either positive or negative energy shifts, despite the limited laser resolution and Doppler broadening effects. 
To allow the incident positron beam to reach the target all electrode voltages (except those applied to T and E1) were initially set to zero, and were then switched on after the beam had passed through using fast high-voltage switches (with $10 \%-90 \%$ voltage rise times of $\approx 25 \mathrm{~ns}$ ). The mean Ps flight time to the first electrode was $\approx 60 \mathrm{~ns}$ and the mean time to reach the end of the guide was on the order of $2 \mu \mathrm{s}$. The $\gamma$-ray signals observed by detectors D2-5 were generated primarily by Ps atoms that were either field ionized or that collided with the vacuum chamber walls after leaving the guide; the latter process is indicated in Fig. 1(a) (see also Sec. IV).

\section{THEORETICAL BACKGROUND}

The Stark effect for Rydberg states of Ps in an electric field $\vec{F}=\left(0,0, F_{z}\right)$ can be treated analytically after solving the field-free Schrödinger equation in parabolic coordinates. Upon such treatment the Stark states into which each unperturbed Rydberg state splits in the presence of the field are characterized by the index $k=n_{1}-n_{2}$, where $n_{1}$ and $n_{2}$ are the parabolic quantum numbers. For each value of $n$, and the azimuthal quantum quantum number $m$, the allowed values of $k$ range from $-(n-|m|-1)$ to $+(n-|m|-1)$ in intervals of 2. The Stark shifts of these states can then be expressed in terms of a series expansion. To second order this gives [48]

$$
\begin{aligned}
E_{\mathrm{Stark}}= & \frac{3}{2} n k e a_{\mathrm{Ps}} F_{z}+\cdots \\
& -\frac{1}{16} n^{4}\left(17 n^{2}-3 k^{2}-9 m^{2}+19\right) \frac{e^{2} a_{\mathrm{Ps}}^{2}}{2 h c R_{\mathrm{Ps}}} F_{z}^{2},
\end{aligned}
$$

where $e$ is the electron charge and $a_{\mathrm{Ps}}$ and $R_{\mathrm{Ps}}$ are the Bohr radius and Rydberg constant corrected for the reduced mass of Ps, respectively. The energy shifts of the $n=13$ Stark states in Ps with $|m|=1$, calculated using this approach, can be seen in Fig. 2. In this figure the energy of each state is displayed for fields up to those in which the electric-field ionization rate of the atom is $10^{10} \mathrm{~s}^{-1}$ [48]. The thicker sections at the high-field end of each curve encompass the range of electric fields for which the ionization rates range from $10^{8}$ to $10^{10} \mathrm{~s}^{-1}$.

The Stark states in Fig. 2 with negative (positive) values of $k$ and hence negative (positive) energy shifts in the electric field exhibit static electric dipole moments oriented parallel (antiparallel) to the field. Consequently, in these states the reduced electron-positron charge density is localized adjacent (opposite) to the Stark saddle point in the potential describing the interaction of the atom with the field [49]. Because of this, states with negative Stark energy shifts ionize in weaker fields than those with positive Stark shifts (see Fig. 2).

A static electric dipole moment, $\vec{\mu}_{\text {elec }}$, can be associated with each Rydberg-Stark state such that [17]

$$
\vec{\mu}_{\text {elec }}=-\frac{3}{2} n k \text { e } a_{\mathrm{Ps}} .
$$

For the $n=13,|k|=11(n=19,|k|=17)$ state this dipole moment has a value of $1100 \mathrm{D}(2500 \mathrm{D})$. In an inhomogeneous electric field, forces can be exerted on Ps atoms prepared in the Stark states in Fig. 2 leading to accelerations

$$
\vec{a}=-\frac{\nabla E_{\text {Stark }}}{M_{\mathrm{Ps}}},
$$

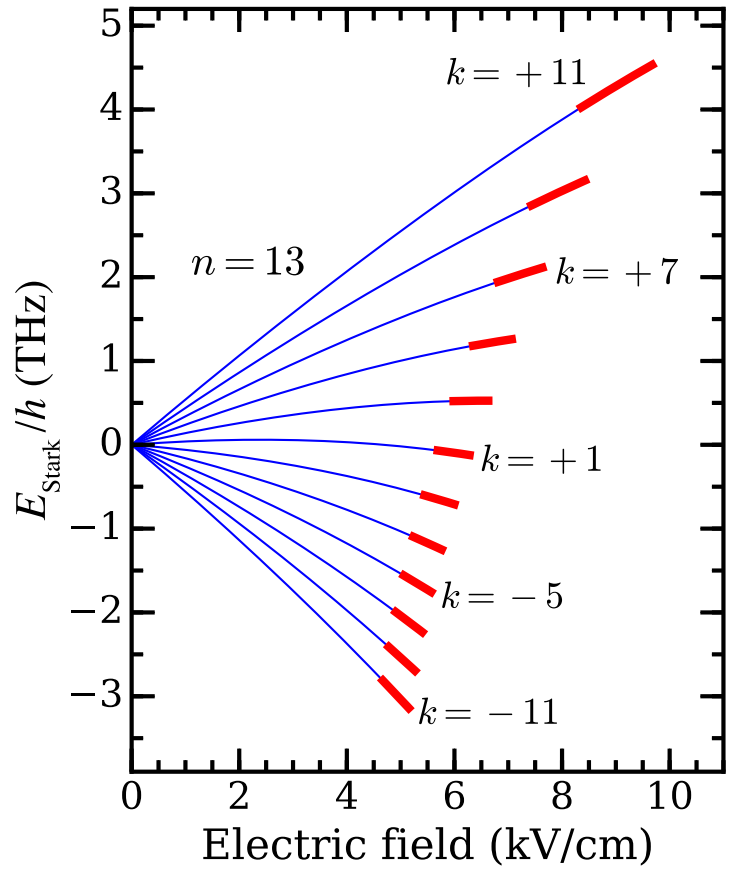

FIG. 2. Energy-level structure of the $n=13$ Stark states in Ps with $|m|=1$. The energy shift of each state, labeled with the index $k$ (see text for details), is displayed over the range of fields in which the ionization rate is $<10^{10} \mathrm{~s}^{-1}$. The thicker sections at the high-field end of each curve denote the fields for which the ionization rate ranges from $10^{8} \mathrm{~s}^{-1}$ to $10^{10} \mathrm{~s}^{-1}$.

where $M_{\mathrm{Ps}}=2 m_{\mathrm{e}}$ is the mass of the Ps atom. Under such conditions atoms in states with negative (positive) Stark energy shifts are accelerated toward regions of stronger (weaker) field, with these states then referred to as high-field seeking (HFS) [low-field seeking (LFS)].

In the experiments reported here, and in the quadrupole guide for Rydberg Ps atoms demonstrated previously $[23,38]$, only atoms in LFS Rydberg-Stark states could be confined and guided in the time-independent inhomogeneous electric fields employed. However, we note that it is possible to simultaneously confine samples in both LFS and HFS Rydberg-Stark states using time-varying electric fields [50]. In the present case atoms in HFS states were accelerated away from the axis of the guide in the radial dimension and towards the regions of high electric-field strength near the electrodes. It is therefore important to ensure that the atoms do not evolve from LFS to HFS states as they travel through the electrode structure. Such evolution, analogous to a Majorana transition in magnetic decelerators or traps [51-53], could occur in atoms that travel through regions in which the electric field changes direction over a short distance and the strength of the field approaches zero. For the electric-field distributions used here these situations occur in the vicinity of the series of electric-field minima located on the axis of the electrode structure within each ring electrode. Such losses are known to occur in other similar Stark deceleration devices (e.g., Refs. [36,54-56]) and the extent to which losses may occur in the case of Ps atoms is not trivially answered owing to their high speeds. 

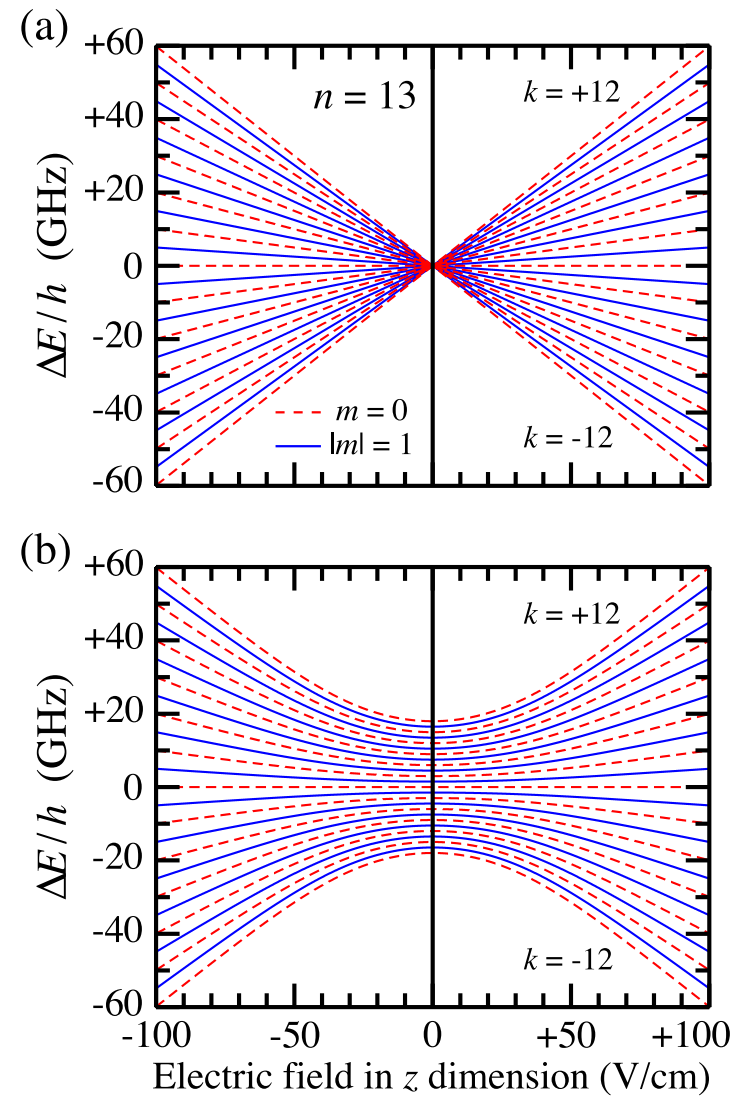

FIG. 3. Stark structure of the $m=0$ (dashed curves) and $|m|=1$ (continuous curves) states in Ps with $n=13$ in an electric field (a) acting only in the $z$ dimension and (b) with an additional component in the $y$ dimension that has a magnitude of $30 \mathrm{~V} / \mathrm{cm}$ when $F_{z}=0 \mathrm{~V} / \mathrm{cm}$.

Rydberg-Stark states in Ps, neglecting fine and hyperfine structure, are degenerate in zero electric field [20] [see Fig. 3(a)]. Because of this, atomic trajectories that pass exactly through a zero field point in the guide may result in the evolution of the atoms from guided LFS states into unguided HFS states. It can be inferred from Fig. 3(a) that, after passing through a zero electric-field point of this kind, an atom prepared in the outermost LFS Stark state with $k=+12$ in a field initially acting in the positive $z$ dimension will evolve into the outermost HFS state with $k=-12$ when the direction of the field reverses. Thus after such a transition the final state populated will possess an electric dipole moment of equal magnitude but opposite orientation in the field to the initial state. To undergo transitions of this kind the atomic trajectories must pass directly through one of the series of zero-field points on the axis of the guide such that an exact crossing of the atomic energy levels occurs. Consequently, transitions of this kind do not occur with high probability.

However, because the Rydberg Ps atoms in the experiments described here travel at high speed $\left(\sim 10^{5} \mathrm{~m} / \mathrm{s}\right)$ through the guiding electric fields, nonadiabatic evolution of the atoms from LFS to HFS states can also occur in the weak-field regions surrounding the zero-field points. In these situations exact crossings of the atomic energy levels are replaced with avoided crossings in the nonzero electric-field minima experienced by the atoms. Thus the field that the atoms travel through does not change direction abruptly but rotates with a residual component at the minimum in the direction perpendicular to that of the initial and final fields. Under these conditions the energy-level structure of the Rydberg-Stark states, when the sign of the $z$ component of the electric field changes, is of the form in Fig. 3(b). In this particular example, the residual component of the electric field in the $y$ dimension is $30 \mathrm{~V} / \mathrm{cm}$ when $F_{z}=0 \mathrm{~V} / \mathrm{cm}$.

To determine, to a first approximation, the conditions under which this second kind of nonadiabatic transition between atomic energy levels occurs under the conditions in which the experiments reported here were performed, two-state LandauZener theory can be employed. Within this approach the probability of diabatic traversal of the avoided crossing at the electric-field minimum can be expressed as [57,58]

$$
\begin{aligned}
P_{\text {diabatic }} & =\exp \left[-\frac{2 \pi\left|W_{k^{\prime} k}\right|^{2}}{\hbar\left(d E_{k^{\prime} k} / d t\right)}\right] \\
& =\exp \left[-\frac{2 \pi\left|W_{k^{\prime} k}\right|^{2}}{\hbar\left(d E_{k^{\prime} k} / d F\right)(d F / d t)}\right],
\end{aligned}
$$

where $d E_{k^{\prime} k} / d t$ is the slew rate through the avoided crossing at the electric-field minimum, with $E_{k^{\prime} k}$ the instantaneous energy separation between the Stark sublevels labeled by indices $k$ and $k^{\prime}$, and for which the minimum energy separation is $E_{\min }=2 W_{k^{\prime} k}$.

Ps Rydberg atoms traveling through the electrode structure of the guide used in the experiments here experience fields which change at a rate of $d F / d t \approx 100 \mathrm{~V} / \mathrm{cm} / \mathrm{ns}$ in the vicinity of the electric-field minima. Because the exponent in Eq. (5) depends on the square of the energy difference between the levels involved in the transition, and only linearly on the inverse of the slew rate, Landau-Zener transitions between Stark sublevels for which the value of $k$ changes by a small amount are more likely to occur than those that result in large changes in $k$. Since the electric field which contributes to the offset from zero must act in the direction perpendicular to fields that dominate at large distances from the minimum, $|\Delta m|=1$ and hence $|\Delta k|=1$ Landau-Zener transitions, for which the values of $W$ in Eq. (5) are smallest, are most probable. Thus for the typical conditions in the experiments reported here nonadiabatic $|\Delta k|=1$ transitions are determined to occur with a probability of greater than 0.5 in electric fields below $10 \mathrm{~V} / \mathrm{cm}$. Since the field gradients around the electric-field minima in the guide are $\sim 10^{4} \mathrm{~V} / \mathrm{cm}^{2}$ the region around the minima in which nonadiabatic LandauZener transitions are likely to occur has a size on the order of $10 \mu \mathrm{m}$. Consequently, despite traveling through the guiding electric fields at high speeds in the experiments, state changing is not expected to contribute significantly to losses of Ps atoms in the type of guide demonstrated here.

\section{PARTICLE TRAJECTORY CALCULATIONS}

Numerical calculations of particle trajectories were performed to characterize the Ps dynamics within the multiring electrode structure shown in Fig. 1(b). The electric-field distributions employed in these calculations were obtained by finite element methods. These calculations were carried 
out for the regions of space within the electrode structure, close to the Ps source, and in the surrounding sections of the vacuum chamber (which forms a grounded boundary). Ps trajectories were calculated numerically using the velocity Verlet algorithm (leapfrog variation) to update the position $\vec{r}_{j}(t)$ and velocity $\vec{v}_{j}(t)$ of the $j$ th atom subject to acceleration $\vec{a}_{j}(t)$ in a time step $\Delta t[59,60]$. The algorithms used had the form

$$
\begin{gathered}
\vec{r}_{j}(t+\Delta t)=\vec{r}_{j}(t)+\vec{v}_{j}(t) \Delta t+\frac{1}{2} \vec{a}_{j}(t) \Delta t^{2}, \\
\vec{v}_{j}(t+\Delta t)=\vec{v}_{j}(t)+\frac{1}{2}\left[\vec{a}_{j}(t)+\vec{a}_{j}(t+\Delta t)\right] \Delta t .
\end{gathered}
$$

In the calculations the acceleration $\vec{a}_{j}$ was determined at each time step from the force exerted on the Ps atom as a result of the Stark shift in the inhomogeneous electric field [see Eq. (3)]. The initial phase-space distributions of the Ps atoms in each calculation were randomly generated by sampling a truncated Maxwell-Boltzmann distribution with a temperature of $800 \mathrm{~K}$, and with a cosine angular distribution. Although the Ps atoms emitted from the target in the experiments were not thermal, this approximation to the speed distribution has been shown to give reasonable agreement with measurements in previous experiments using nominally identical mesoporous film targets [43,49]. For each case of interest 15000 Ps trajectories were calculated. The trajectories were terminated if the atoms collided with material objects in the vacuum system, decayed by fluorescence, or encountered sufficiently strong electric fields to cause ionization.

Calculations were performed for atoms in states with $n=13$ and $n=19$. These Rydberg states were chosen to allow two different types of experiments to be performed. At $n=13$ LFS states could be selectively prepared by laser photoexcitation but the atoms could not be detected by electricfield ionization at the end of the guide because of the limited field strength that could be generated in this location. On the other hand, in the experiments performed at $n=19$ the LFS or HFS character of the Stark states could not be completely controlled at the time of laser photoexcitation but atoms in these states could be detected by electric-field ionization at the end of the guide. The calculations were all performed for atoms in outer Stark states with $|k|=n-2$ and account for the radiative lifetimes of the Rydberg Ps atoms which are $\approx 5$ and $25 \mu$ s for $n=13$ and $n=19$, respectively, assuming only $|m|=1$ states are produced [23].

Figure 4 shows calculated Ps trajectories for the field configurations corresponding to $V_{\text {odd }}=-4 \mathrm{kV}$ and $V_{\text {even }}=0 \mathrm{~V}$ [excluding E1; see Fig. 1(b)] for $n=13$ LFS and HFS states. These results indicate that, with the guide off (i.e., odd and even electrodes set to $0 \mathrm{~V}$ ), few atoms reach the end of the electrode structure. When the guide is on no HFS atoms reach the end of the electrode structure as they are deflected away from the axis of the apparatus by the inhomogeneous electric field. Atoms in LFS states are transported to the end of the guide, as expected.

When atoms leave the guide structure they enter into an electric field determined by the potential on the last electrode and the geometry of the surrounding vacuum chamber. This can be seen in Fig. 5, which shows calculated trajectories for atoms in Rydberg-Stark states with $n=13$ and $n=19$ for
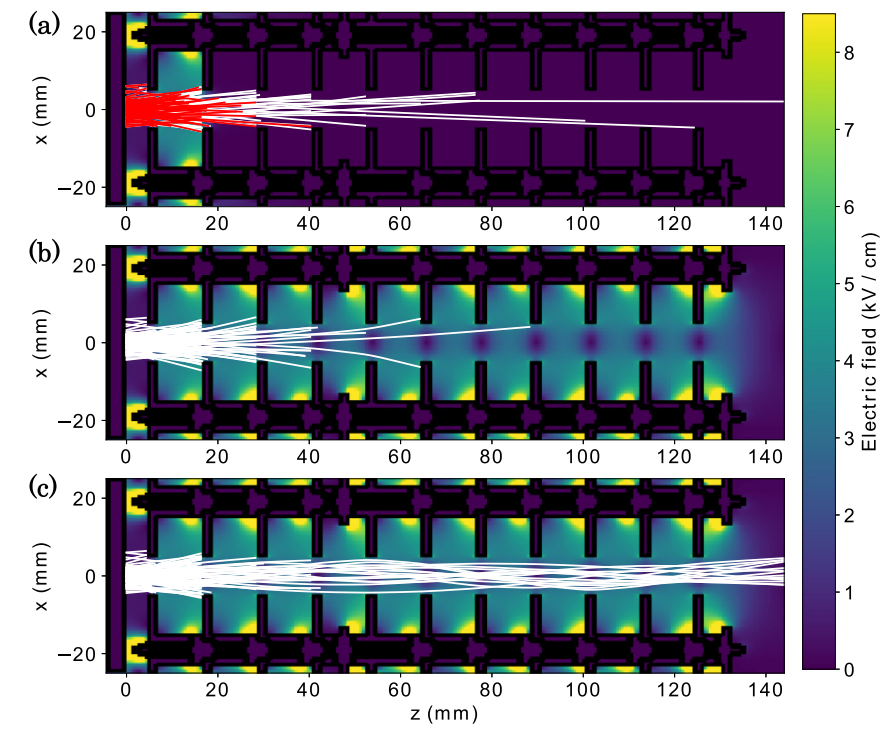

FIG. 4. Calculated Ps trajectories for (a) atoms in the $n=13$, $k=+11$ LFS state with the guide off, (b) atoms in the $n=13, k=$ -11 HFS state with the guide on, and (c) atoms in the $n=13$, $k=+11$ LFS state with the guide on. When the guide was off all electrodes (except $\mathrm{T}$ and $\mathrm{E} 1$ ) were set to $0 \mathrm{~V}$. When the guide was on $V_{\text {odd }}=-4 \mathrm{kV}$ and $V_{\text {even }}=0 \mathrm{~V}$. The darker (red) trajectories in (a) correspond to atoms in the ground state which have a mean annihilation lifetime of 142 ns. 100 atoms were propagated in each of these calculations.

which the electric fields at the end of the guide lie below and above the threshold for electric-field ionization, respectively. Under these conditions $n=13$ atoms will be detected primarily following fluorescence to the ground state after exiting the guide and collisions with the vacuum chamber walls. Conversely, all $n=19$ atoms will be field ionized and are subsequently detected via the annihilation of liberated positrons which are attracted to the last electrode. These different processes give rise to markedly different TOF spectra, measurements of which are shown in Sec. V.

\section{RESULTS AND DISCUSSION}

The goal of the experiments described here was to evaluate the efficacy with which a multiring electrode structure can be used to control the motion of Ps atoms in Rydberg-Stark states with large electric dipole moments, and in particular to evaluate the role of nonadiabatic transitions between these states in the vicinity of the electric-field minima inherent in such a device. This work builds upon previous guiding experiments employing quadrupole electric fields [37], but the current arrangement is potentially much more versatile since deceleration and trapping of Ps atoms can in principle be achieved if time-varying fields are applied to the electrodes [27]. However, this will only be possible if the zero-field regions present in the multiring structure do not result in catastrophic losses, as discussed in Sec. III. Measurements were performed with Ps atoms in Rydberg-Stark states with low $(n=13)$ and high $(n=19)$ values of the principal quantum number in order to evaluate different aspects of the multiring electrode structure. 


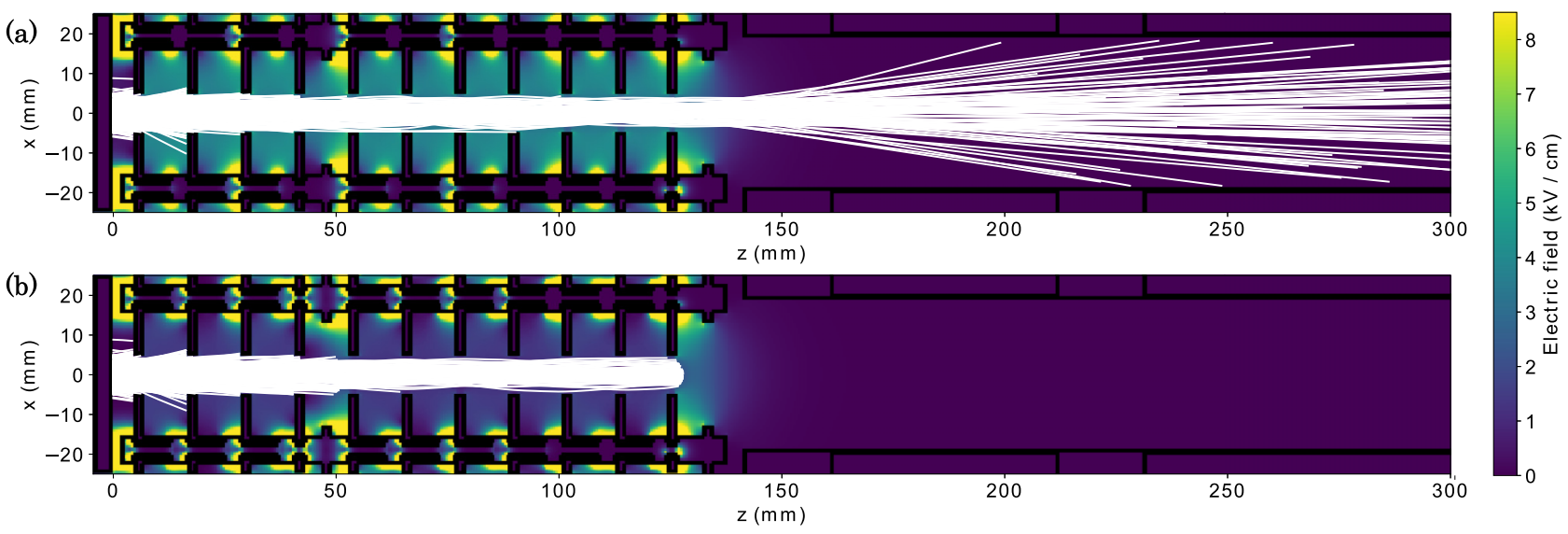

FIG. 5. Calculated trajectories of Ps atoms in LFS states with (a) $n=13$ and $k=+11$ and (b) $n=19$ and $k=+17$. The applied guiding potentials were (a) $V_{\text {odd }}=-4 \mathrm{kV}$ and $V_{\text {even }}=0 \mathrm{kV}$ and (b) $V_{\text {odd }}=-4 \mathrm{kV}$ and $V_{\text {even }}=-2.5 \mathrm{kV}$. The electric fields experienced by the $n=13$ atoms lie below the electric-field ionization threshold, whereas $n=19$ atoms are fully ionized after the last electrode (E11). The trajectories of atoms that are not field ionized eventually strike the vacuum chamber walls where they annihilate. 500 atoms were propagated in each of these calculations.

(i) Low $n$ atoms $(n=13)$ were used to study the relative guiding efficiency of selected Stark states (i.e., LFS and HFS states) because for these atoms it was possible to lift the degeneracy of the Stark states at the time of laser photoexcitation with an applied electric field and then tune the IR laser wavelength to predominantly select particular Stark states [24]. This was not possible using higher $n$ states, for which the corresponding required fields caused Stark manifolds with consecutive values of $n$ to overlap.

(ii) High $n$ atoms $(n=19)$ were used to facilitate efficient detection by electric-field ionization [49] at the end of the guide, thereby making the TOF profiles sharper and more representative of the Ps motion in the electrode structure, as opposed to the broader profiles obtained through detection upon collisions with the vacuum chamber walls. Detection by electric-field ionization was not possible for lower $n$ states, for which ionization requires much larger electric fields than can be applied in the present apparatus.

Rydberg Ps atoms with $n=13$ were prepared in a mean electric field of $\sim 300 \mathrm{~V} / \mathrm{cm}$. However, we note that this field was not uniform because of the open design of the electrodes. The target electrode bias [see Fig. 1(a)] was used to control the positron beam implantation energy and thus the energy of emitted Ps atoms [47]. For all experiments using atoms photoexcited to states with $n=13$ the potentials applied to the target, T, and E1 were -4.25 and $-4.0 \mathrm{kV}$, respectively, whereas for experiments at $n=19$ the potential applied to T was $-4.0 \mathrm{kV}$. The potentials applied to the other electrodes were varied according to the experimental requirements.

The measured Stark-broadened spectrum of the $2{ }^{3} P_{J} \rightarrow$ $13^{3} S / 13^{3} D$ transitions is shown in Fig. 6(a). These data were recorded via single-shot lifetime methods [40] using detector D1 and are characterized by the parameter $S_{\gamma}$ as described in Sec. II. The spectral resolution of our measurements was $\approx 200 \mathrm{GHz}$, limited by Doppler broadening and the bandwidth of the excitation lasers. A single Gaussian function representative of this spectral resolution is indicated by the curve with a maximum of 0.3 centered at a wavelength of $\lambda \approx 746.7 \mathrm{~nm}$ in Fig. 6(a). The relative strengths of electric dipole transitions to the individual Stark states are also shown in this figure as the vertical bars. Also shown is the calculated spectral intensity distribution, obtained following the convolution of

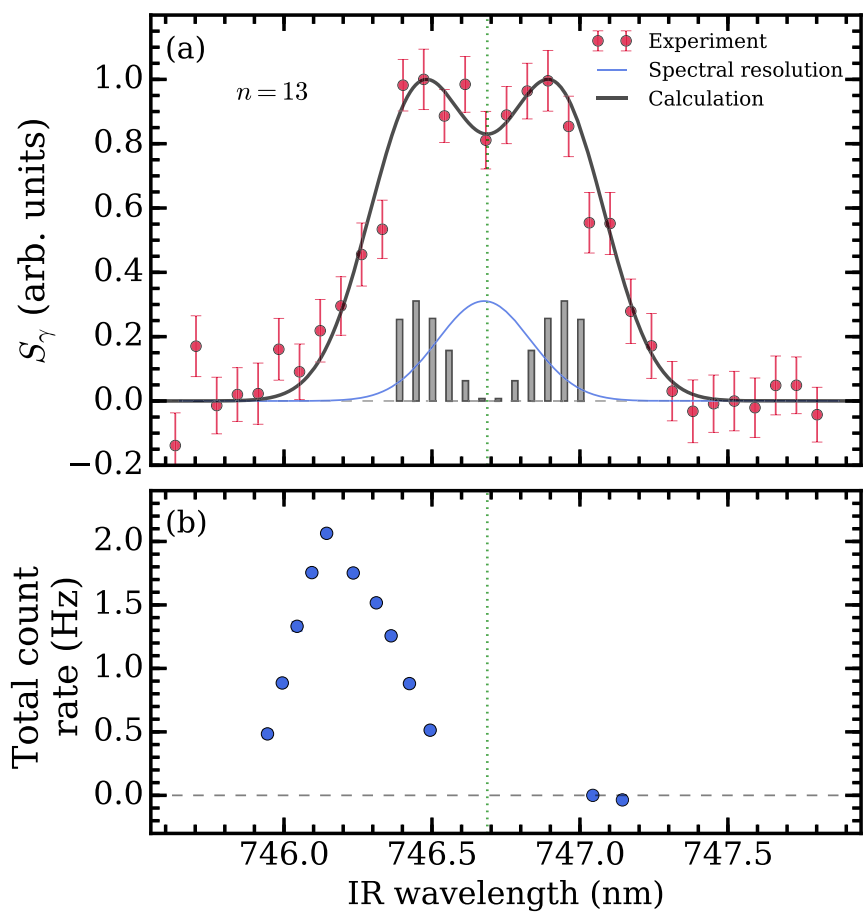

FIG. 6. (a) Spectrum of $2{ }^{3} P_{J} \rightarrow 13{ }^{3} \mathrm{~S} / 13{ }^{3} \mathrm{D}$ transitions in an electric field of $\sim 300 \mathrm{~V} / \mathrm{cm}$. (b) The corresponding total guided atom count rate for $V_{\text {odd }}=-4 \mathrm{kV}$ and $V_{\text {even }}=0$. The bars in (a) represent the calculated spectral positions and relative spectral intensities of transitions to the individual $n=13$ Stark states. The thin (blue) Gaussian curve represents an effective spectral resolution of $200 \mathrm{GHz}$. The thick (black) continuous curve results from the convolution of the calculated transition moments with the spectral resolution function. The error bars in (b) are smaller than the symbols. 


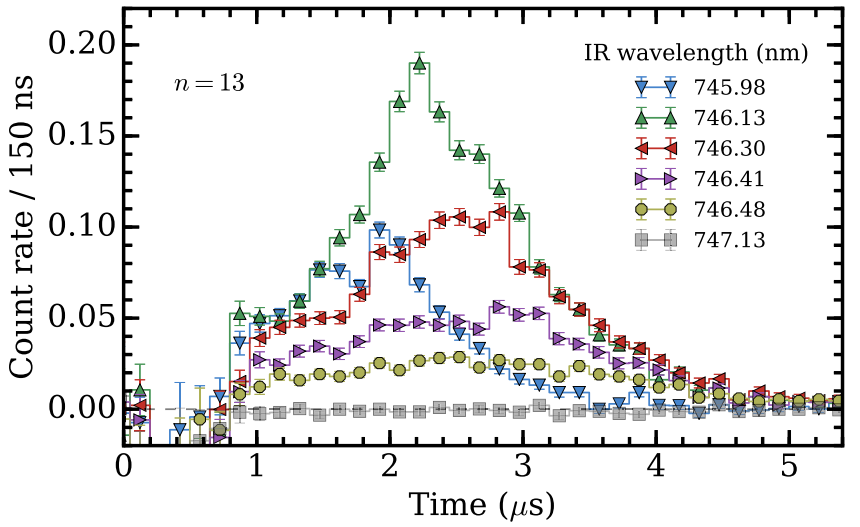

FIG. 7. TOF profiles recorded for different wavelengths of the IR laser for $n=13$ Rydberg Ps atoms. The guide potentials used for these measurements were $V_{\text {odd }}=-4 \mathrm{kV}$ and $V_{\text {even }}=0 \mathrm{kV}$.

these transition intensities with a Gaussian spectral resolution function.

When guiding electric fields were applied to the electrode structure, atoms prepared in LFS states were transported through the device and subsequently observed using detectors D2-5 [see Fig. 1(a)] in single-event counting mode [23], as described in Sec. II. The total count rate of transported atoms is shown in Fig. 6(b) as a function of the laser wavelength. These data confirm that the LFS Stark states with larger dipole moments (i.e., the outer states on the short wavelength side of the spectrum) are more efficiently transported [37]. A selection of the corresponding TOF profiles are shown in Fig. 7. From the analysis of the probability and effects of Landau-Zener transitions described in Sec. III nonadiabatic Ps dynamics of this kind are not expected to significantly affect the measured guiding efficiencies in these data.

Additional TOF profiles, obtained using a fixed IR laser wavelength and different guiding fields, are shown in Fig. 8(a). Here $n=13$ LFS (HFS) atoms were photoexcited by tuning the laser to a wavelength of $\lambda=$ 746.22 (746.91) $\mathrm{nm}$; it is again clear from these data that HFS states were not guided. The maximum field gradients in the device were limited by the potentials that could be applied to the high-voltage switches $(4 \mathrm{kV})$, so for some measurements a positive bias was applied to the even numbered electrodes. The "guide potential" indicated in the figures refers to the potential difference between adjacent guide electrodes; the corresponding electric-field distributions are of the form shown in Fig. 4.

As is evident from Fig. 8, stronger electric fields, and hence field gradients, result in more Ps atoms being guided. This is consistent with the effects observed in the data presented in Fig. 7, where the guiding efficiency increases when atoms with larger dipole moments are prepared. The small observed decrease at the highest fields is due to a small reduction in the detection efficiency (see Fig. 5). For stronger guiding fields the divergence of the Ps beam is lower, and more atoms are able to travel out of the field of view of the detectors before annihilation. Indeed, with the addition of extended electrodes simulations suggest that a stronger focusing effect is possible if time-varying fields are used to account for the different Ps
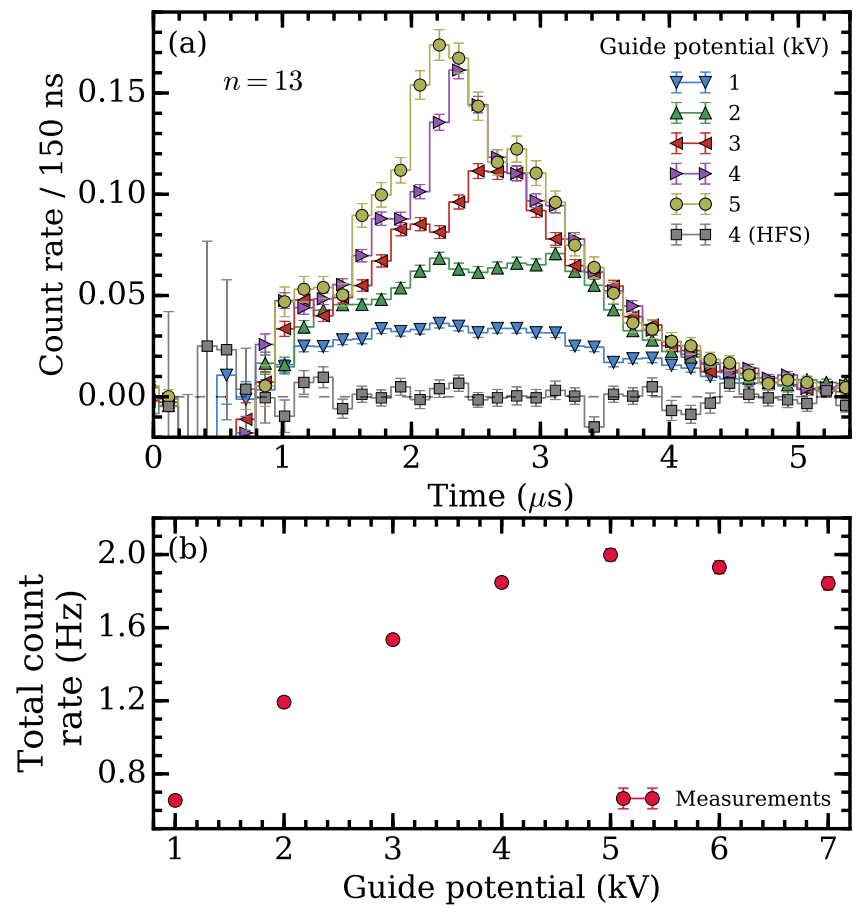

FIG. 8. (a) TOF spectra for $n=13$ Ps with the laser tuned to produce LFS or HFS states as described in the text and (b) the total count rate measured for different applied fields. The guide potentials indicated in (a) refer to the voltage difference between alternate electrodes. The error bars in (b) are smaller than the symbols.

velocities [61]. This could be useful in experiments in which Ps atoms interact with other species in order to study scattering or charge exchange mechanisms [62-64]. We note that it is difficult to characterize the overall efficiency and acceptance of the guide because we do not accurately know the initial phase-space properties of the Ps atoms. In future experiments involving thermal Ps sources with known distributions, and which can be imaged, it will be possible to characterize and improve the guide acceptance.

TOF profiles of the type shown in Fig. 8 represent the intrinsic velocity distribution of the Ps atoms, modified by the acceptance of the guide. When atoms are detected via annihilation following fluorescence, or collisions with the walls of the vacuum chamber beyond the end of the guide, such profiles are further spread out, owing to the variations in path length. However, if the atoms are field ionized after leaving the electrode structure, liberated positrons will be rapidly accelerated into E11 and annihilate, resulting in a sharper TOF profile. Calculated Ps trajectories for both of these scenarios are shown in Fig. 5, and calculated and measured TOF profiles representing these cases are shown in Fig. 9. In recording these data Ps atoms excited to $n=19$ were guided using electric fields generated by applying potentials of $V_{\text {odd }}=-4 \mathrm{kV}$ and $V_{\text {even }}=-2.5 \mathrm{kV}$. This was sufficient to guide $n=19$ LFS states. The electric field present after E11 was large enough to ionize all $n=19$ atoms but none of the $n=13$ atoms.

The mean arrival time of the $n=13$ atoms shown in Fig. 9 is later than that of the $n=19$ atoms, owing to the longer average flight path. The shape of these TOF profiles is determined 


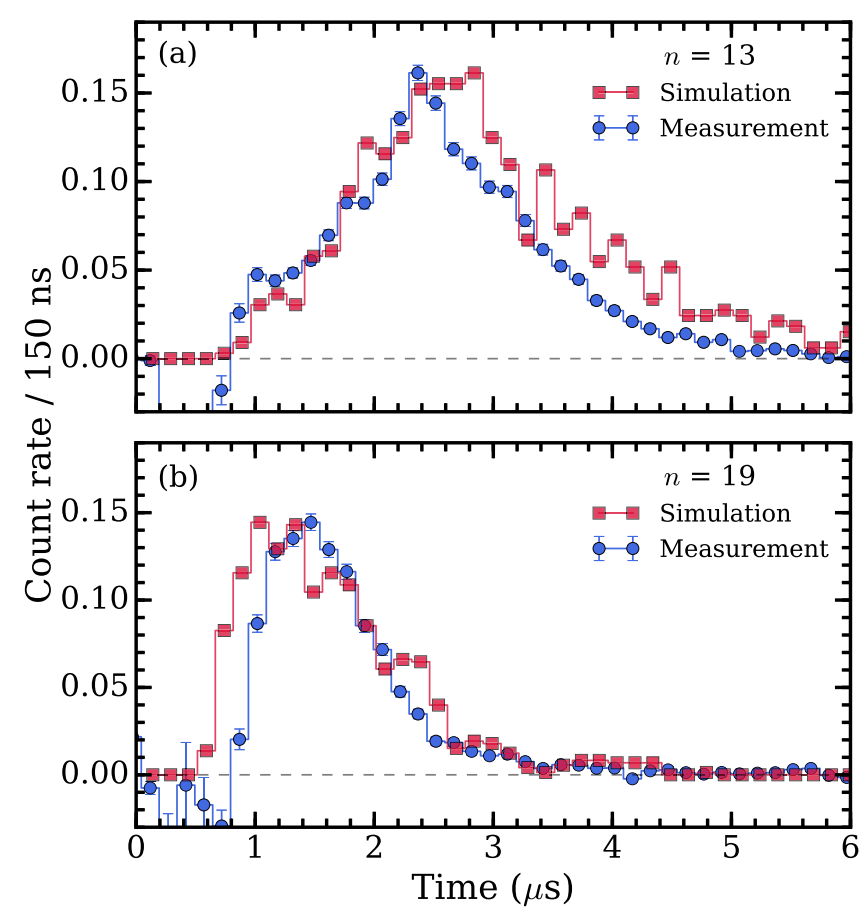

FIG. 9. Calculated and measured TOF distributions for (a) $n=$ 13 and (b) $n=19$ Ps atoms. The calculated data use the same guiding fields as the measurements and the Ps phase-space distributions described in Sec. IV. 15000 trajectories were calculated in each case with the count rates then scaled to match the experimental data.

by the Ps distributions as well as the dynamics of the guiding and detection processes. As a result the profiles cannot be described by simple analytic functions, and the widths are therefore not well defined. Nevertheless, the $n=13$ profile is clearly broader in time than that measured by electric-field ionization. The calculated TOF profiles shown in Fig. 9 are in reasonable agreement with the measured data.

Here we have exploited the different detection modes that can be implemented in our experiments to record TOF spectra to validate our simulations and evaluate the operation of the multiring guide. However, Ps TOF measurements can also be used to provide information related to Ps production mechanisms [65-71] or the intrinsic properties of materials from which Ps atoms are emitted [72-74]. If such measurements are performed using ground-state atoms, then the obtainable resolution is fundamentally limited by the relatively short Ps lifetime. By exciting Ps atoms to long-lived Rydberg states, however, it is possible to significantly improve this resolution $[11,75]$.

Ps atoms are generally emitted from porous films (or other similar materials, e.g., Ref. [76]) with a broad spread of velocities [77]. Atoms with different velocities can therefore be selectively excited simply by adjusting the time delay between the excitation laser pulse and the incident positron pulse [43]. The fraction of Ps atoms optically excited for different laser delays, as quantified by the parameter $S_{\gamma}$ (see Sec. II) is shown in Fig. 10(a). This fraction varies according to the Ps temporal emission profile, modified by the velocity dependent excitation efficiency (faster atoms are excited less efficiently). As the laser delay is scanned, a broad Ps emission
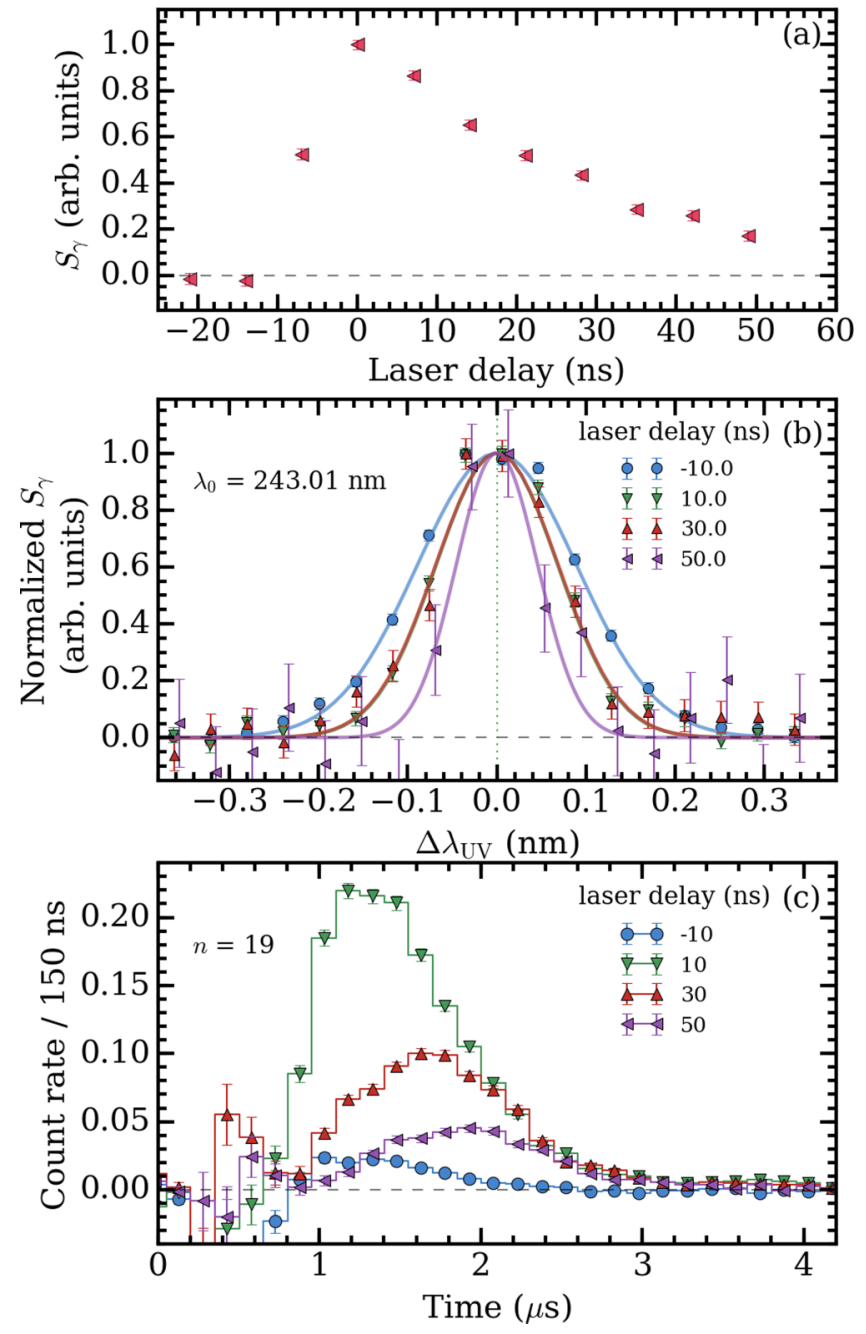

FIG. 10. (a) Ps excitation fraction parameter $S_{\gamma}$ measured for different laser delays relative to the positron implantation time, (b) Doppler profiles, and (c) TOF spectra, measured for the same laser delays. The Ps TOF profiles shown in (c) were recorded using $n=19$ atoms. The guide potentials used for these measurements were $V_{\text {odd }}=-4 \mathrm{kV}$ and $V_{\text {even }}=-2.5 \mathrm{kV}$, meaning that the atoms were field ionized at the final electrode (see Fig. 5).

time window of $\approx 50 \mathrm{~ns}$ is observed, determined predominantly by the Ps flight time through the spatial extent of the laser beams.

Figure 10(b) shows Doppler broadened $1{ }^{3} S_{1} \rightarrow 2{ }^{3} P_{J}$ spectra, measured for different laser delays. As discussed in Sec. II, these measurements can be used to determine the Ps rms velocity spread in the transverse direction (i.e., the direction of the UV laser). We determine the Ps longitudinal speeds from TOF distributions, as shown in Fig. 10(c). Figure 11 shows the Ps kinetic energies determined from the Doppler and TOF data presented in Fig. 10. These measurements are consistent with each other, although the good agreement is at least partially coincidental since, even though the underlying longitudinal and transverse velocity components are expected to be comparable [43], the Doppler profiles are sensitive to both the Ps speed and angular distributions, whereas the guide has a related, but different, phase-space acceptance. 


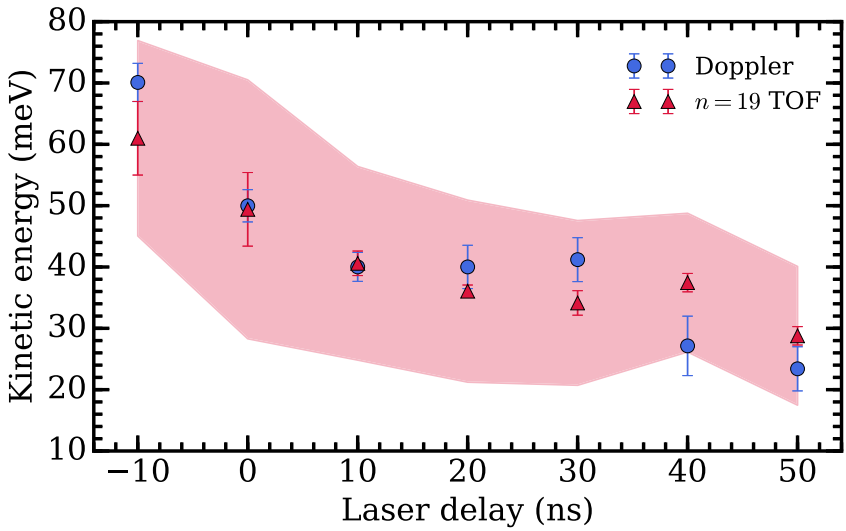

FIG. 11. Kinetic energy of Ps atoms photoexcited at a range of laser time delays, as determined from the Doppler broadening spectroscopy and TOF measurements shown in Fig. 10. The shaded band represents the total Ps energy spread as observed in the TOF profiles, while the error bars on these data points are estimates of the mean energies obtained by fitting Gaussian functions to the TOF profiles. The error bars on the Doppler broadening data were derived from the Gaussian widths of the measured line shapes.

\section{CONCLUSIONS}

We have shown that a multiring electrode structure can be used to guide Ps atoms in LFS Rydberg-Stark states with large static electric dipole moments. Since this type of structure necessarily has regions of zero electric field through which the LFS atoms may travel, the possibility of nonadiabatic transitions to unguided states exists, which could lead to a loss of atoms. By considering the Ps atom trajectories and the associated Landau-Zener dynamics, we have shown that in general this is not a significant loss mechanism under the conditions for which the experiments were performed. Our results therefore indicate that similar electrode structures can be employed for more advanced experiments in which the individual electrodes are addressed using time-varying potentials, making it possible to generate traveling electric traps in which to transport, decelerate, and trap Ps atoms, or to decelerate atoms by rapidly switching off applied field gradients after atoms have slowed down by moving in these gradients [27].

The physical electrode structure described in this work can be used for such experiments; the main modifications required are the addition of more complex time-dependent voltage control sequences that can be applied to all of the electrodes. We anticipate that, just by using more HV switches, slower Ps atoms can be trapped in the device, and work is ongoing to determine the optimal voltage pulse sequences required for deceleration. This will depend on the Ps properties and is likely to require $\approx \mathrm{kV}$ pulses that can be switched in different sequences on a $\mu$ s time scale (e.g., Ref. [36]) and is therefore within the capabilities of commercially available $\mathrm{HV}$ switches. For slower Ps atoms excited to higher values of $n$, lower voltage pulses $(150 \mathrm{~V})$ generated by fast $(5 \mathrm{MHz})$ $\mathrm{HV}$ amplifiers, which are also commercially available, may be more efficacious (e.g., Ref. [34]). It is also possible to find a different balance between voltage and timing properties using homemade electronics (e.g., Ref. [78]). Decelerating and focusing Rydberg Ps atoms to form beams over which complete phase-space control could be realized would significantly advance the precision of Ps optical [79] and microwave [80] spectroscopy, which has languished behind the theoretical uncertainties for decades [81] and shows no signs of catching up as theory continues to advance [82].

More optimal Ps atom sources are also necessary for new Ps measurements, such as a determination of the Rydberg constant [83]. There exist many techniques in optical spectroscopy that have not been applied to Ps or have not achieved high precision because of the properties of Ps. For example, saturated absorption spectroscopy (SAS) can provide accurate Doppler free measurements [84], but in the case of Ps the Doppler profiles are so broad that it is difficult to obtain any signal [85]. The availability of slower atoms focused or collimated into a narrow beam would therefore open up many new areas of Ps spectroscopy. Well-known techniques (including SAS) could then be employed [86], and it may be possible to generate useful signals using narrow-band lasers [87]. Measurements using the Ramsay separated oscillatory field method [88], or variations [89], with Rydberg Ps could help shed some light on the proton radius puzzle [90-92], and would be complimentary to measurements using other Rydberg systems where the proton influence is reduced [93-95]. It is worth pointing out that more sophisticated excitation schemes, such as Doppler free or chirped pulse excitation, would not be beneficial with the present hot Ps since we would then simply generate more hot atoms that would not be guided. This again emphasizes the need for improved Ps sources.

Finally, decelerated Rydberg Ps beams are also potentially useful for some gravity free-fall measurements [96,97], which would require Ps atoms to fly for tens of ms. At typical speeds (i.e., $10^{7} \mathrm{~cm} / \mathrm{s}$ ) this would require an impractically long flight path. Moreover, to perform such an experiment it would also be necessary to transfer a significant fraction of the Rydberg population into circular states [98], which is challenging for Ps [49] and would also be much more feasible using a slow Ps source.

\section{ACKNOWLEDGMENTS}

The authors are grateful to L. Liszkay for providing mesoporous silica samples. This work was supported by the EPSRC under Grant No. EP/R006474/1.
[1] J. Pérez-Ríos and S. T. Love, Searching for light dark matter through positronium decay, Eur. Phys. J. D 72, 44 (2018).
[2] S. G Karshenboim, Precision study of positronium: Testing bound state QED theory, Int. J. Mod. Phys. A 19, 3879 (2004). 
[3] S. G. Karshenboim, Precision Physics of Simple Atoms and Constraints on a Light Boson with Ultraweak Coupling, Phys. Rev. Lett. 104, 220406 (2010).

[4] H. Lamm, p-state positronium for precision physics: An ultrafine splitting at $\alpha^{6}$, Phys. Rev. A 96, 022515 (2017).

[5] M. S. Safronova, D. Budker, D. DeMille, D. F. J. Kimball, A. Derevianko, and C. W. Clark, Search for new physics with atoms and molecules, Rev. Mod. Phys. 90, 025008 (2018).

[6] J. Jaeckel and A. Ringwald, The low-energy frontier of particle physics, Annu. Rev. Nucl. Part. Sci. 60, 405 (2010).

[7] A. Signer, Low-energy precision physics and the high-energy frontier, Phys. Proc. 51, 25 (2014).

[8] D. B. Cassidy, Experimental progress in positronium laser physics, Eur. Phys. J. D 72, 53 (2018).

[9] A. P. Mills, Jr., Thermal activation measurement of positron binding energies at surfaces, Solid State Commun. 31, 623 (1979).

[10] L. Liszkay, C. Corbel, P. Perez, P. Desgardin, M. F. Barthe, T. Ohdaira, R. Suzuki, P. Crivelli, U. Gendotti, A. Rubbia, M. Etienne, and A. Walcarius, Positronium reemission yield from mesostructured silica films, Appl. Phys. Lett. 92, 063114 (2008).

[11] A. C. L. Jones, H. J. Goldman, Q. Zhai, P. Feng, H. W. K. Tom, and A. P. Mills, Jr., Monoenergetic Positronium Emission from Metal-Organic Framework Crystals, Phys. Rev. Lett. 114, 153201 (2015).

[12] G. Scholes, Atomic and Molecular Beam Methods (Oxford University Press, New York, 1988), Vol. 1.

[13] S. Berko and H. N. Pendleton, Positronium, Annu. Rev. Nucl. Part. Sci. 30, 543 (1980).

[14] P. A. M. Dirac, On the annihilation of electrons and protons, Math. Proc. Cambridge Philos. Soc. 26, 361 (1930).

[15] A. Ore and J. L. Powell, Three-Photon Annihilation of an Electron-Positron Pair, Phys. Rev. 75, 1696 (1949).

[16] M. S. Fee, S. Chu, A. P. Mills, Jr., R. J. Chichester, D. M. Zuckerman, E. D. Shaw, and K. Danzmann, Measurement of the positronium $1{ }^{3} S_{1}-2{ }^{3} S_{1}$ interval by continuous-wave twophoton excitation, Phys. Rev. A 48, 192 (1993).

[17] T. F. Gallagher, Rydberg Atoms (Cambridge University Press, Cambridge, UK, 1994).

[18] A. I. Alekseev, Two-photon annihilation of positronium in the P-state, Sov. Phys. JETP 7, 826 (1958).

[19] A. I. Alekseev, Three-photon annihilation of positronium in the P-state, Sov. Phys. JETP 9, 1312 (1959).

[20] H. A. Bethe and E. E. Salpeter, Quantum Mechanics of Oneand Two-Electron Atoms (Springer, Berlin, 1957).

[21] A. Osterwalder and F. Merkt, Using High Rydberg States as Electric Field Sensors, Phys. Rev. Lett. 82, 1831 (1999).

[22] F. Merkt and R. N. Zare, On the lifetimes of Rydberg states probed by delayed pulsed field ionization, J. Chem. Phys. 101, 3495 (1994).

[23] A. Deller, A. M. Alonso, B. S. Cooper, S. D. Hogan, and D. B. Cassidy, Measurement of Rydberg positronium fluorescence lifetimes, Phys. Rev. A 93, 062513 (2016).

[24] T. E. Wall, A. M. Alonso, B. S. Cooper, A. Deller, S. D. Hogan, and D. B. Cassidy, Selective Production of Rydberg-Stark States of Positronium, Phys. Rev. Lett. 114, 173001 (2015).

[25] W. H. Wing, Electrostatic Trapping of Neutral Atomic Particles, Phys. Rev. Lett. 45, 631 (1980).
[26] T. Breeden and H. Metcalf, Stark Acceleration of Rydberg Atoms in Inhomogeneous Electric Fields, Phys. Rev. Lett. 47, 1726 (1981).

[27] S. D. Hogan, Rydberg-Stark deceleration of atoms and molecules, EPJ Tech. Instrum. 3, 1 (2016).

[28] D. Townsend, A. L. Goodgame, S. R. Procter, S. R. Mackenzie, and T. P. Softley, Deflection of krypton Rydberg atoms in the field of an electric dipole, J. Phys. B 34, 439 (2001).

[29] E. Vliegen and F. Merkt, Normal-Incidence Electrostatic Rydberg Atom Mirror, Phys. Rev. Lett. 97, 033002 (2006).

[30] E. Vliegen, P. A. Limacher, and F. Merkt, Measurement of the three-dimensional velocity distribution of Stark-decelerated Rydberg atoms, Eur. Phys. J. D 40, 73 (2006).

[31] P. Lancuba and S. D. Hogan, Guiding Rydberg atoms above surface-based transmission lines, Phys. Rev. A 88, 043427 (2013).

[32] P. Allmendinger, J. Deiglmayr, J. A. Agner, H. Schmutz, and F. Merkt, Surface-electrode decelerator and deflector for Rydberg atoms and molecules, Phys. Rev. A 90, 043403 (2014).

[33] S. D. Hogan, P. Allmendinger, H. Saßmannshausen, H. Schmutz, and F. Merkt, Surface-electrode Rydberg-Stark Decelerator, Phys. Rev. Lett. 108, 063008 (2012).

[34] P. Lancuba and S. D. Hogan, Transmission-line decelerators for atoms in high Rydberg states, Phys. Rev. A 90, 053420 (2014).

[35] S. D. Hogan and F. Merkt, Demonstration of ThreeDimensional Electrostatic Trapping of State-Selected Rydberg Atoms, Phys. Rev. Lett. 100, 043001 (2008).

[36] C. Seiler, S. D. Hogan, H. Schmutz, J. A. Agner, and F. Merkt, Collisional and Radiative Processes in Adiabatic Deceleration, Deflection and Off-Axis Trapping of a Rydberg Atom Beam, Phys. Rev. Lett. 106, 073003 (2011).

[37] A. Deller, A. M. Alonso, B. S. Cooper, S. D. Hogan, and D. B. Cassidy, Electrostatically Guided Rydberg Positronium, Phys. Rev. Lett. 117, 073202 (2016).

[38] A. M. Alonso, B. S. Cooper, A. Deller, L. Gurung, S. D. Hogan, and D. B. Cassidy, Velocity selection of Rydberg positronium using a curved electrostatic guide, Phys. Rev. A 95, 053409 (2017).

[39] A. C. L. Jones, J. Moxom, H. J. Rutbeck-Goldman, K. A. Osorno, G. G. Cecchini, M. Fuentes-Garcia, R. G. Greaves, D. J. Adams, H. W. K. Tom, A. P. Mills, and M. Leventhal, Focusing of a Rydberg Positronium Beam with an Ellipsoidal Electrostatic Mirror, Phys. Rev. Lett. 119, 053201 (2017).

[40] B. S. Cooper, A. M. Alonso, A. Deller, T. E. Wall, and D. B. Cassidy, A trap-based pulsed positron beam optimised for positronium laser spectroscopy, Rev. Sci. Instrum. 86, 103101 (2015).

[41] A. M. Alonso, B. S. Cooper, A. Deller, and D. B. Cassidy, Single-shot positron annihilation lifetime spectroscopy with LYSO scintillators, Nucl. Instrum. Methods Phys. Res., A 828, 163 (2016).

[42] L. Liszkay, M. F. Barthe, C. Corbel, P. Crivelli, P. Desgardin, M. Etienne, T. Ohdaira, P. Perez, R. Suzuki, V. Valtchev, and A. Walcarius, Orthopositronium annihilation and emission in mesostructured thin silica and silicalite-1 films, Appl. Surf. Sci. 255, 187 (2008).

[43] A. Deller, B. S. Cooper, T. E. Wall, and D. B. Cassidy, Positronium emission from mesoporous silica studied by laser-enhanced time-of-flight spectroscopy, New J. Phys. 17, 043059 (2015). 
[44] K. P. Ziock, R. H. Howell, F. Magnotta, R. A. Failor, and K. M. Jones, First Observation of Resonant Excitation of High- $n$ States in Positronium, Phys. Rev. Lett. 64, 2366 (1990).

[45] D. B. Cassidy, T. H. Hisakado, H. W. K. Tom, and A. P. Mills, Jr., Efficient Production of Rydberg Positronium, Phys. Rev. Lett. 108, 043401 (2012).

[46] D. B. Cassidy, S. H. M. Deng, H. K. M. Tanaka, and A. P. Mills, Jr., Single shot positron annihilation lifetime spectroscopy, Appl. Phys. Lett. 88, 194105 (2006).

[47] D. B. Cassidy, P. Crivelli, T. H. Hisakado, L. Liszkay, V. E. Meligne, P. Perez, H. W. K. Tom, and A. P. Mills, Jr., Positronium cooling in porous silica measured via Doppler spectroscopy, Phys. Rev. A 81, 012715 (2010).

[48] R. J. Damburg and V. V. Kolosov, Theoretical studies of hydrogen Rydberg atoms in electric fields, in Rydberg States of Atoms and Molecules, edited by R. F. Stebbings and F. B. Dunning (Cambridge University Press, Cambridge, UK, 1983), pp. 31-72.

[49] A. M. Alonso, L. Gurung, B. A. D. Sukra, S. D. Hogan, and D. B. Cassidy, State-selective electric-field ionization of Rydberg positronium, Phys. Rev. A 98, 053417 (2018).

[50] A. Deller and S. D. Hogan, Confinement of High- and LowField-Seeking Rydberg Atoms Using Time-Varying Inhomogeneous Electric Fields, Phys. Rev. Lett. 122, 053203 (2019).

[51] E. Majorana, Atomi orientati in campo magnetico variabile, Nuovo Cimento (1924-1942) 9, 43 (1932).

[52] A. L. Migdall, J. V. Prodan, W. D. Phillips, T. H. Bergeman, and H. J. Metcalf, First Observation of Magnetically Trapped Neutral Atoms, Phys. Rev. Lett. 54, 2596 (1985).

[53] S. D. Hogan, D. Sprecher, M. Andrist, N. Vanhaecke, and F. Merkt, Zeeman deceleration of $\mathrm{H}$ and D, Phys. Rev. A 76, 023412 (2007).

[54] T. E. Wall, S. K. Tokunaga, E. A. Hinds, and M. R. Tarbutt, Nonadiabatic transitions in a Stark decelerator, Phys. Rev. A 81, 033414 (2010).

[55] S. A. Meek, G. Santambrogio, B. G. Sartakov, H. Conrad, and G. Meijer, Suppression of nonadiabatic losses of molecules from chip-based microtraps, Phys. Rev. A 83, 033413 (2011).

[56] D. Reens, H. Wu, T. Langen, and J. Ye, Controlling spin flips of molecules in an electromagnetic trap, Phys. Rev. A 96, 063420 (2017).

[57] L. D. Landau, Zur theorie der energieübertragung ii, Phys. Z. Sowjetunion 2, 46 (1932).

[58] C. Zener, Non-adiabatic crossing of energy levels, Proc. R. Soc. London, Ser. A 137, 696 (1932).

[59] M. E. Tuckerman, Statistical Mechanics: Theory and Molecular Simulation (Oxford University Press, Oxford, UK, 2010), pp. 100-101.

[60] R. J. Sadus, Molecular Simulation of Fluids: Theory, Algorithms and Object-Orientation (Elevier, Oxford, UK, 2002), pp. 228229.

[61] D. B. Cassidy and S. D. Hogan, Atom control and gravity measurements using Rydberg positronium, Int. J. Mod. Phys.: Conf. Ser. 30, 1460259 (2014).

[62] A. R. Swann, D. B. Cassidy, A. Deller, and G. F. Gribakin, Formation of positron-atom bound states in collisions between Rydberg Ps and neutral atoms, Phys. Rev. A 93, 052712 (2016).

[63] M. Charlton, A. S. Kadyrov, and I. Bray, Heating due to momentum transfer in low-energy positronium-antiproton scattering, Phys. Rev. A 94, 032701 (2016).
[64] W. A. Bertsche, M. Charlton, and S. Eriksson, Cold neutral atoms via charge exchange from excited state positronium: A proposal, New J. Phys. 19, 053020 (2017).

[65] S. M. Curry and A. L. Schawlow, Measurements of the kinetic energy of free positronium formed in $\mathrm{MgO}$, Phys. Lett. A 37, 5 (1971).

[66] A. P. Mills, Jr. and L. Pfeiffer, Velocity spectrum of positronium thermally desorbed from an Al(111) surface, Phys. Rev. B 32, 53 (1985).

[67] R. H. Howell, I. J. Rosenberg, and M. J. Fluss, Production of energetic positronium at metal surfaces, Phys. Rev. B 34, 3069 (1986).

[68] P. Sferlazzo, S. Berko, and K. F. Canter, Time-of-flight spectroscopy of positronium emission from quartz and magnesium oxide, Phys. Rev. B 35, 5315 (1987).

[69] Y. Nagashima, Y. Morinaka, T. Kurihara, Y. Nagai, T. Hyodo, T. Shidara, and K. Nakahara, Origins of positronium emitted from $\mathrm{SiO}_{2}$, Phys. Rev. B 58, 12676 (1998).

[70] P. Crivelli, U. Gendotti, A. Rubbia, L. Liszkay, P. Perez, and C. Corbel, Measurement of the orthopositronium confinement energy in mesoporous thin films, Phys. Rev. A 81, 052703 (2010).

[71] P. Crivelli, D. Cooke, B. Barbiellini, B. L. Brown, J. I. Feldblyum, P. Guo, D. W. Gidley, L. Gerchow, and A. J. Matzger, Positronium emission spectra from self-assembled metal-organic frameworks, Phys. Rev. B 89, 241103(R) (2014).

[72] R. H. Howell, I. J. Rosenberg, M. J. Fluss, R. E. Goldberg, and R. B. Laughlin, Positronium time-of-flight spectroscopy of dissimilar metals, Phys. Rev. B 35, 5303 (1987).

[73] A. P. Mills, Jr., L. Pfeiffer, and P. M. Platzman, Positronium Velocity Spectroscopy of the Electronic Density of States at a Metal Surface, Phys. Rev. Lett. 51, 1085 (1983).

[74] A. P. Mills, Jr., E. D. Shaw, R. J. Chichester, and D. M. Zuckerman, Search for adiabatic positronium emission from a metal surface, Phys. Rev. B 40, 8616 (1989).

[75] A. C. L. Jones, H. J. Rutbeck-Goldman, T. H. Hisakado, A. M. Piñeiro, H. W. K. Tom, A. P. Mills, Jr., B. Barbiellini, and J. Kuriplach, Angle-Resolved Spectroscopy of Positronium Emission from a $\mathrm{Cu}(110)$ Surface, Phys. Rev. Lett. 117, 216402 (2016).

[76] C. G. Fischer, M. H. Weber, C. L. Wang, S. P. McNeil, and K. G. Lynn, Positronium in low temperature mesoporous films, Phys. Rev. B 71, 180102(R) (2005).

[77] D. B. Cassidy, T. H. Hisakado, V. E. Meligne, H. W. K. Tom, and A. P. Mills, Jr., Delayed emission of cold positronium from mesoporous materials, Phys. Rev. A 82, 052511 (2010).

[78] A. Osterwalder, S. A. Meek, G. Hammer, H. Haak, and G. Meijer, Deceleration of neutral molecules in macroscopic traveling traps, Phys. Rev. A 81, 051401(R) (2010).

[79] M. S. Fee, A. P. Mills, Jr., S. Chu, E. D. Shaw, K. Danzmann, R. J. Chichester, and D. M. Zuckerman, Measurement of the Positronium $1{ }^{3} S_{1}-2{ }^{3} S_{1}$ Interval by Continuous-Wave TwoPhoton Excitation, Phys. Rev. Lett. 70, 1397 (1993).

[80] D. Hagena, R. Ley, D. Weil, G. Werth, W. Arnold, and H. Schneider, Precise Measurement of $n=2$ Positronium FineStructure Intervals, Phys. Rev. Lett. 71, 2887 (1993).

[81] R. Ley, Atomic physics of positronium with intense slow positron beams, Appl. Surf. Sci. 194, 301 (2002).

[82] G. S. Adkins, Higher order corrections to positronium energy levels, J. Phys.: Conf. Ser. 1138, 012005 (2018). 
[83] F. Biraben, Spectroscopy of atomic hydrogen, Eur. Phys. J.: Spec. Top. 172, 109 (2009).

[84] T. W. Hänsch, I. S. Shahin, and A. L. Schawlow, HighResolution Saturation Spectroscopy of the Sodium $d$ Lines with a Pulsed Tunable Dye Laser, Phys. Rev. Lett. 27, 707 (1971).

[85] D. B. Cassidy, T. H. Hisakado, H. W. K. Tom, and A. P. Mills, Jr., Positronium Hyperfine Interval Measured Via Saturated Absorption Spectroscopy, Phys. Rev. Lett. 109, 073401 (2012).

[86] W. Demtröder, Laser Spectroscopy, 3rd ed. (Springer, New York, 2003).

[87] N. Kolachevsky, J. Alnis, S. D. Bergeson, and T. W. Hänsch, Compact solid-state laser source for $1 S-2 S$ spectroscopy in atomic hydrogen, Phys. Rev. A 73, 021801(R) (2006).

[88] N. F. Ramsey, Experiments with separated oscillatory fields and hydrogen masers, Rev. Mod. Phys. 62, 541 (1990).

[89] K. Kato, T. D. G. Skinner, and E. A. Hessels, UltrahighPrecision Measurement of the $n=2$ Triplet $P$ Fine Structure of Atomic Helium Using Frequency-Offset Separated Oscillatory Fields, Phys. Rev. Lett. 121, 143002 (2018).

[90] A. Beyer, L. Maisenbacher, A. Matveev, R. Pohl, K. Khabarova, A. Grinin, T. Lamour, D. C. Yost, T. W. Hänsch, N. Kolachevsky, and T. Udem, The Rydberg constant and proton size from atomic hydrogen, Science 358, 79 (2017).

[91] H. Fleurbaey, S. Galtier, S. Thomas, M. Bonnaud, L. Julien, F. Biraben, F. Nez, M. Abgrall, and J. Guéna, New Measurement of the $1 S-3 S$ Transition Frequency of Hydrogen: Contribution to the Proton Charge Radius Puzzle, Phys. Rev. Lett. 120, 183001 (2018).

[92] M. Kalinowski, Deuteron charge radius from the Lamb-shift measurement in muonic deuterium, Phys. Rev. A 99, 030501(R) (2019).

[93] J. C De Vries, Ph.D. thesis, Massachusetts Institute of Technology, 2001.

[94] A. Ramos, K. Moore, and G. Raithel, Measuring the Rydberg constant using circular Rydberg atoms in an intensitymodulated optical lattice, Phys. Rev. A 96, 032513 (2017).

[95] N. Hölsch, M. Beyer, E. J. Salumbides, K. S. E. Eikema, W. Ubachs, C. Jungen, and F. Merkt, Benchmarking Theory with an Improved Measurement of the Ionization and Dissociation Energies of $\mathrm{H}_{2}$, Phys. Rev. Lett. 122, 103002 (2019).

[96] A. P. Mills, Jr. and M. Leventhal, Can we measure the gravitational free fall of cold Rydberg state positronium? Nucl. Instrum. Methods Phys. Res., B 192, 102 (2002).

[97] G. Dufour, D. B. Cassidy, P. Crivelli, P. Debu, A. Lambrecht, V. V. Nesvizhevsky, S. Reynaud, A. Y. Voronin, and T. E. Wall, Prospects for studies of the free fall and gravitational quantum states of antimatter, Adv. High Energy Phys. 2015, 379642 (2015).

[98] R. G. Hulet and D. Kleppner, Rydberg Atoms in "Circular" States, Phys. Rev. Lett. 51, 1430 (1983). 